Review Article

\title{
Extrahepatic Autoimmune Diseases in Patients with Autoimmune Liver Diseases: A Phenomenon Neglected by Gastroenterologists
}

\author{
Liping Guo, ${ }^{1}$ Lu Zhou, ${ }^{1}$ Na Zhang, ${ }^{2}$ Baoru Deng, ${ }^{1}$ and Bangmao Wang ${ }^{1}$ \\ ${ }^{1}$ Department of Gastroenterology and Hepatology, Tianjin Medical University General Hospital, Tianjin 300052, China \\ ${ }^{2}$ Department of Rheumatology, Tianjin Medical University General Hospital, Tianjin 300052, China \\ Correspondence should be addressed to Lu Zhou; zhou_lu@126.com and Bangmao Wang; bmwang0926@outlook.com
}

Received 20 June 2016; Accepted 3 November 2016; Published 16 January 2017

Academic Editor: Paolo Gionchetti

Copyright (C) 2017 Liping Guo et al. This is an open access article distributed under the Creative Commons Attribution License, which permits unrestricted use, distribution, and reproduction in any medium, provided the original work is properly cited.

\begin{abstract}
Autoimmune liver diseases (AILDs) often coexist with other extrahepatic autoimmune diseases (EHAIDs). The spectrum of EHAIDs in patients with AILDs is similar, whereas the incidence is different. Notably, autoimmune thyroid disease and Sjogren's syndrome are the most common EHAIDs. Associated extrahepatic diseases may predate the appearance of AILDs or coincide with their onset. More frequently, they may appear during the course and even occur years after the diagnosis of AILDs. Importantly, associated EHAIDs may influence the natural course and prognosis of AILDs. To date, a definite pathophysiological pathway which contributes to the coexistence of AILDs and EHAIDs is still lacking. The current view of autoimmunity clustering involves a common susceptibility genetic background which applies to related pathologies. Herein, we review the current published researches regarding EHAIDs in patients with AILDs, particularly in relation to their clinical impact and pathophysiology. In managing patients with AILDs, gastroenterologists should be aware of the possibly associated EHAIDs to ensure a prompt diagnosis and better outcome.
\end{abstract}

\section{Introduction}

Autoimmune liver diseases (AILDs) fall into two broad categories, those with hepatic predominance such as autoimmune hepatitis (AIH) and those with predominance of cholestatic features such as primary biliary cirrhosis $(\mathrm{PBC})$ and primary sclerosing cholangitis (PSC). The concurrence of clinical, biochemical, serological, and/or histological features suggesting $\mathrm{AIH}$ and $\mathrm{PBC}$ (or PSC) has been described as overlap syndrome (OS). Importantly, AILDs also coexist with other extrahepatic autoimmune diseases (EHAIDs) and conditions, thereby causing not only liver damage, but also extrahepatic injury. However, the relationship between AILDs and concomitant EHAIDs is strongly debated. Currently, two hypotheses exist: (i) it is thought that AILDs are part of multiple organ involvement in a systemic autoimmune disease, particularly in non-organ specific autoimmune diseases such as systemic lupus erythematosus (SLE), rheumatoid arthritis (RA), systemic sclerosis (SSc), and Sjogren's syndrome (SS); (ii) it is thought that AILDs and concomitant extrahepatic autoimmune conditions are different disorders, linked by a common pathogenetic pathway, for example, the coexistence of AILDs and chronic lymphocytic thyroiditis, hyperthyroidism, myasthenia gravis, or pernicious anemia. Herein, we discuss EHAIDs in patients with AILDs, particularly in relation to their clinical impact and pathophysiology.

\section{The Spectrum of EHIADs in Patients with AILDs}

The spectrum of EHAIDs in patients with AILDs is similar in current reports, whereas the incidence is different (Table 1). Notably, autoimmune thyroid disease (AITD) and SS are the most common EHAIDs in patients with AILDs. Associated extrahepatic diseases may predate the appearance of AILDs or coincide with their onset. More frequently, they may appear during the course and even occur years after the 


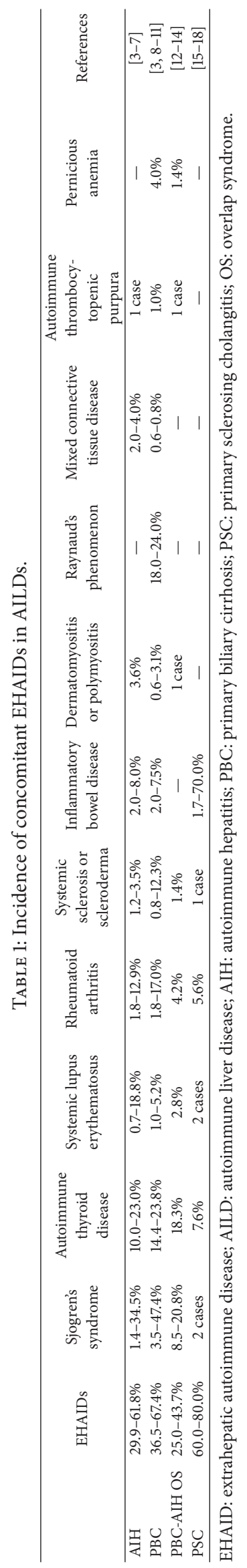


TABLE 2: Liver involvement in CTDs.

\begin{tabular}{lccccc}
\hline & Liver involvement & AIH & PBC & PSC & References \\
\hline Systemic lupus erythematosus & $3.0-79.0 \%$ & $2.7-20.0 \%$ & $2.7-15.0 \%$ & 1 case & {$[1,19-29]$} \\
Sjogren's syndrome & $7.0-49.0 \%$ & $6.0-47.0 \%$ & $35.0-57.0 \%$ & 11 cases & {$[22,29-31]$} \\
Systemic sclerosis or scleroderma & $1.1 \%$ & 11 cases & $51.2 \%$ & $51.2 \%$ & {$[22,29,32-34]$} \\
Antiphospholipid syndrome & - & 5 cases & 1 case & 1 case & {$[13,35-37]$} \\
Dermatomyositis or polymyositis & - & $7.1 \%$ & $14.3 \%$ & - & {$[13,38]$} \\
\hline
\end{tabular}

AIH: autoimmune hepatitis; PBC: primary biliary cirrhosis; PSC: primary sclerosing cholangitis.

diagnosis of AILDs. Watt et al. [1] reported that 84 of 160 (53\%) PBC patients had at least one additional extrahepatic autoimmune condition, and 16 of 37 (43\%) patients developed thyroid diseases prior to the detection of $\mathrm{PBC}$, while 19 of 37 (51\%) patients detected thyroid diseases at the same time or following the diagnosis of PBC; 10 of 12 (83\%) patients developed sclerosis symptoms prior to the detection of PBC. Another report also showed that the onset time of AIH and dermatomyositis (DM) was uneven [2]. While explanations for the discrepancies in incidence and onset time are lacking, different geographical and genetic backgrounds in studies may be involved.

\section{Nonspecific Liver Involvement or AILDs in the Course of EHIADs}

Autoimmunity clustering frequently increases the difficulty of diagnosis. It is clinically important for gastroenterologists to early screen patients with AILDs for concomitant EHIADs and to make an accurate diagnosis according to their respective diagnostic criteria. Connective tissue diseases (CTDs) are systemic disorders that have an autoimmune basis and involve multiple organs or tissues, such as liver, kidney, and lung. Indeed, patients with CTDs often have concomitant liver abnormalities; about $3.0-79.0 \%$ of patients with SLE and 7.0-49.0\% of patients with SS showed liver dysfunction (Table 2). When confronted with such patients, gastroenterologists need to classify these liver abnormalities as a primary liver disease with associated autoimmune, clinical, and laboratory features or a generalized liver involvement manifestation of CTDs. The classical example of this differential diagnosis dilemma is AIH and CTDs associated hepatitis, both of which have autoimmune symptoms. Antinuclear antibody (ANA) and immunoglobulin (Ig) G are not unique in AIH, which can be also positive in CTDs. On the other hand, patients with CTDs often show liver abnormalities as mentioned before. Therefore, it is big confusion to distinguish them just according to symptoms, physical signs, and autoantibodies. Liver biopsy may be helpful in such patients. Histopathological manifestations of CTDs associated hepatitis may vary from subclinical liver diseases with nonspecific changes to chronic active hepatitis, chronic persistent hepatitis, fibrosis, cirrhosis, nodular regenerative hyperplasia, and so on [17, 21].

Otherwhile, external factors such as drugs can trigger susceptible patients with risk alleles of the major histocompatibility complex (MHC). Drug-induced autoimmune liver disease (DIAILD) refers to the latent autoimmunity with positive autoantibodies, including drug-induced liver injury (DILI), drug-induced-AIH (DI-AIH), and immunity mediated DILI (IM-DILI) [39]. It is important to remember that another main cause of biochemical liver abnormalities in patients with CTDs is drug-induced alterations. Almost all drugs in the armamentarium against SLE or other rheumatologic diseases may lead to liver toxicity, such as nonsteroidal anti-inflammatory drugs (NSAIDs). NSAIDassociated liver injuries vary from slightly biochemical and histological abnormalities to severe liver fibrosis, cirrhosis, chronic liver failure, or even fulminant hepatic failure [22, 23]. A prospective study found that 28 of 260 (10.8\%) patients with active SLE showed salicylate poisoning and biochemical liver abnormalities. In this study, 14 patients underwent liver biopsies, by which a nonspecific inflammatory reaction was confirmed. Additionally, immunosuppressive drugs such as antitumor necrosis factor- (TNF-) $\alpha$ were reported to induce liver injuries. The immune-mediated drug reaction in the liver must be monitored during using biologics [40]. Histological performances of AIH and DILI have certain similarities, including interface hepatitis, inflammatory cells infiltration in portal area, and centrilobular 3 zone necrosis [17, 41]. Suzuki et al. [42] compared 35 cases of DILI with 28 cases of AIH according to Ishak score, portal inflammatory cell types, penetration phenomenon, rosette, and cholestasis. The results showed that interface hepatitis, focal necrosis, and portal inflammation existed both in AIH and DILI; however, neutrophils infiltration in portal area and cholestasis were more common in DILI. In addition, Suzuki et al. [42] suggested that compared with AIH DILI had no obvious liver fibrosis. Other studies indicated that eosinophils infiltration was more common in DILI, but some findings showed that eosinophils infiltration was not conducive to distinguish DILI from AIH [31]. Therefore, in addition to medication history and clinical manifestations, liver biopsy is crucial to assist in distinguishing AILDs in the course of EHAIDs from CTDs associated or drug-induced liver injuries.

\section{The Impact of Concomitant EHAIDs on the Natural Course and Prognosis of AILDs}

Whether the concomitant EHAIDs affect the natural course and prognosis of AILDs is unclear as only a few related researches have been carried out. In a latest study from UK, Wong et al. [6] systematically assessed features and clinical impact of EHAID on AIH. Autoimmune skin diseases were more prevalent in AIH-2 than AIH-1 (21.9\% versus 7.0\%, 
$P=0.009$ ), which suggested that presence of EHAIDs might influence clinical phenotype of $\mathrm{AIH}$ at presentation. Personal history of EHAIDs was more commonly found in AIH patients with than without first-degree family history of EHAIDs [48/86 (55.8\%) versus 169/446 (37.9\%), $P=0.002$ ]. AIH patients with EHAIDs were more often women $(85.2 \%$ versus $76.1 \%, P=0.008)$, had higher posttreatment IAIHG score (22 versus 20, $P<0.001$ ), had less reactivity to smooth muscle antibodies (49.8\% versus $65.0 \%, P<0.001)$, were more likely to have mild fibrosis at diagnosis $(20.9 \%$ versus $6.5 \%, P<0.001)$, and less often had ascites (6.3\% versus $13.6 \%, P=0.008)$ and coagulopathy (1.18 versus $1.27, P=$ 0.013 ) at presentation. However, presence of EHAIDs did not significantly affect disease progression, prognosis, and survival in AIH.

Muratori et al. [43] investigated 608 Italian patients with AILDs (327 with AIH and 281 with PBC) for concomitant EHAIDs and assessed the incidence and clinical impact of associated EHAIDs on AILDs. AIH patients with EHAIDs showed significant female predominance (male/female: 63/ 163 versus 9/91). In addition, an EHAID was more often detected in patients with an onset of AIH devoid of any particular liver-related symptoms, and the concomitant EHAIDs did not modify the features of PBC patients.

Wang et al. [19] screened 322 Chinese PBC patients for the presence of CTDs and identified the differences in clinical features and laboratory findings between $\mathrm{PBC}$ patients with or without CTDs. Compared to patients with PBC alone, PBC-SLE patients had lower $\gamma$-glutamyl transpeptidase $(\gamma$ GGT) and immunoglobulin M (IgM) levels, suggesting that presence of SLE in PBC patients appeared to be associated with significantly less extensive liver damage and SLE might protect against progression of $\mathrm{PBC}$ by delaying cirrhosis and the need for liver transplantation; PBC-RA patients had higher serum immunoglobulin G (IgG) and alkaline phosphatase (ALP) levels, suggesting that presence of RA might be a harmful factor in the prognosis of $\mathrm{PBC}$. The presence of SS, SSc, and polymyositis (PM) did not seem to have any impact on the clinical course and prognosis of $\mathrm{PBC}$; however, their unique features emerged. PBC-SS patients were more likely to have fever and elevated erythrocyte sedimentation rate (ESR), a higher incidence of rheumatoid factor (RF) seropositivity, and interstitial lung disease (ILD), suggesting that patients with concomitant autoimmune disorders might have an aggravated inflammatory response; $\mathrm{PBC}$-SSc patients had a higher incidence of ILD; PBC-PM patients had a higher white blood cell (WBC) count and incidence of myocardial involvement. In another study, adjusting for sex, age, log bilirubin, and ALP, the risk of transplantation or death from diagnosis was significantly lower in PBC-SSc (hazard ratio $0.116, P=0.01$ ) [44], suggesting that PBC patients with SSc had a better prognosis.

As mentioned before, the first two researches investigated the features and clinical impact of concomitant EHAIDs on AIH patients. Similar result was female predominance in $\mathrm{AIH}$ patients with concomitant EHAIDs. Besides analyses of some clinical features at AIH presentation and prognosis, Wong et al. [6] paid more attention to the personal and family EHAIDs history and impact of EHAIDs on AIH clinical phenotype.
The other three researches all analyzed the clinical features and prognosis of PBC patients with or without concomitant EHAIDs. Wang et al. [19] found that SLE or RA might be a beneficial or harmful factor in the prognosis of PBC, and SS, SSc, and PM seemed to have no impact on the clinical course and prognosis of PBC. However, the last paper suggested that concomitant SSc significantly lowered the risk of transplantation or death in PBC patients. Taken together, different EHAIDs may have different influences on the natural course and prognosis of AILDs. Certain EHAIDs may aggravate the systemic inflammatory response and liver damage, and others may alleviate the liver inflammatory response, consequently achieving a better prognosis. Meanwhile, it is worth paying more attention to the higher incidence of ILD in autoimmune clustering. Of course, differences between the researches may be due to entirely different population backgrounds. Furthermore, some multicenter researches with a large sample size and different population background are needed to explore and verify these findings.

\section{Common Pathophysiological Pathways in AILDs and EHAIDs}

Autoimmune diseases appear to result from a complex series of interactions between susceptibility genes, environment, and immune system. However, the researches on pathophysiological pathways of concomitant autoimmune diseases are still relatively limited. Recently, with the development of molecular genetics, human genome-wide association studies (GWAs) and risk-associated single nucleotide polymorphism (SNP) have revealed that these patterns of coexistence/overlap depend predominantly on genetic determinants [24-26, 32, 33, 35-38]. Most of these so-called susceptible gene loci are widely distributed in many autoimmune diseases and thus contribute strongly to their coexistence. The recently completed GWAs reported a significant connection between PBC and STAT4, which is also the prominent risk gene in AITD, type 1 diabetes, SLE, RA, SS, and inflammatory bowel disease (IBD) $[24,32,36,45]$. Notably, the most commonly recorded connection between MHC and SSc was the HLA-B8, a DR3-containing haplotype. Interestingly, a high frequency of HLA-DR3 detected in PSC patients was also noted. Similarly, HLA-DRB1, HLA-DRB3, and HLADR4 have been suggested to contribute to the coexistence of PSC and IBD [46-49]. In addition, HLA-DR2, HLA-DR3, and IRF5 have been reported as the common susceptibility genes in both PBC and SS $[25,26]$, further indicating that a common genetic background contributes to the coexistence of AILDs and other extrahepatic autoimmune disorders.

The coexistence of SS and PBC is a major example of the so-called autoimmune clustering, and the underlying mechanism has been relatively well studied. Both of these autoimmune diseases are characterized by the progressive immune-mediated destruction of epithelial tissues, either in salivary and lacrimal glands or in the intrahepatic bile ducts. Antimitochondrial autoantibodies (AMAs) to the E2 subunit of the pyruvate dehydrogenase complex (PDC-E2) are serological hallmarks of $\mathrm{PBC}$, which were detected in 
95\% of patients with PBC [50-52]. Surprisingly, the PBC autoantigen, PDC-E2, has been demonstrated to be present on the surface of salivary epithelial cells in the salivary glands of PBC patients with SS. Matsumoto et al. [53] found that SS and AILD had a similar immune and inflammatory response, especially $\mathrm{CD}^{+} \mathrm{T}$ cells in related organizations, suggesting that the liver and salivary glands, lacrimal glands, or other secreting glands may have the same antigenicity. It is plausible to predict that on the basis of a similar susceptibility gene background environmental triggers (putatively infectious agents and xenobiotics) cause salivary or biliary epithelial cell apoptosis and immune tolerance breakdown to selfantigens which are not protected by PDC-E2, leading to the cellular immune response with predominant $\mathrm{CD}^{+} \mathrm{T}$ cell infiltration and the mucosal immune response mediated by $\operatorname{IgA}[25,26,33,38]$. Therefore, common susceptibility gene backgrounds are involved in the pathophysiological pathways between PBC and SS.

Last but not least, autoimmune polyendocrine syndrome type 1 (APS-1) should be mentioned. It is a rare monogenetic recessive disorder caused by mutations in the autoimmune regulator (Aire) gene. Patients with APS-1 always developed SS, anemia, diabetes, alopecia, vitiligo, gastritis, and $\mathrm{AIH}$, the last one affecting up to $20 \%$ of APS-1 patients [54], which provides a possible connection between AILD and some endocrine-associated autoimmune diseases. To summarize, common susceptibility genes, environmental triggers, and antigen cross-reaction cooperatively contribute to a possibly shared pathogenesis which is involved in the coexistence of AILDs and EHAIDs.

\section{Conclusion}

Commonly, more than one autoimmune condition can occur in same AILD patient. Herein, we discuss EHAIDs in patients with AILDs, particularly in relation to the clinical impact and pathophysiology of these diseases. The incidence of EHAIDs in AILDs and onset time of them can be different. These discrepancies might be explained by different geographical and genetic backgrounds between studies. Importantly, autoimmunity clustering frequently increases the difficulty of diagnosis. In particular, biochemical liver abnormalities in patients with CTDs are common, which may be the result of previous treatments with potentially hepatotoxic drugs or CTDs associated nonspecific liver involvement. Liver biopsy is crucial in distinguishing AILDs in the course of EHAIDs from CTDs associated or drug-induced liver injuries. In our review, it is worth mentioning that we first summarize and analyze a few researches regarding clinical features and impact of concomitant EHAIDs on AILDs. When overlapping with other extrahepatic autoimmune diseases, patients with AILDs manifest special clinical and laboratory features which may have an effect on the natural course and prognosis of AILDs. Certain EHAIDs may aggravate the systemic inflammatory response and liver damage, and others may alleviate the liver inflammatory response, consequently achieving a better prognosis. Furthermore, some multicenter researches with a large sample size and different population background are needed to explore and verify these findings. Finally, we try to explore the pathophysiological pathway which contributes to the coexistence of AILDs and EHAIDs. Currently, it is widely recognized that a common susceptibility genetic background involves the autoimmunity clustering. Therefore, in managing patients with AILDs, gastroenterologists should be aware of concomitant EHAIDs to ensure a prompt diagnosis and better outcome. Future researches should pay more attention to the relationship between genomics and immune regulator factors and confirm a common and distinct pathway involved in the pathogeneses of autoimmunity clustering.

\section{Competing Interests}

The authors declare that they have no competing interests.

\section{Authors' Contributions}

Liping Guo wrote the paper; Lu Zhou, Na Zhang, Baoru Deng, and Bangmao Wang designed and reviewed the manuscript.

\section{Acknowledgments}

This work is supported by the National Natural Science Foundation of China (Grant no. 81200282 and no. 81470834).

\section{References}

[1] F. E. Watt, O. F. W. James, and D. E. J. Jones, "Patterns of autoimmunity in primary biliary cirrhosis patients and their families: a population-based cohort study," QJM, vol. 97, no. 7, pp. 397-406, 2004.

[2] C. Pamfil, E. Candrea, E. Berki, H. I. Popov, P. I. Radu, and S. Rednic, "Primary biliary cirrhosis-autoimmune hepatitis overlap syndrome associated with dermatomyositis, autoimmune thyroiditis and antiphospholipid syndrome," Journal of Gastrointestinal and Liver Diseases, vol. 24, no. 1, pp. 101-104, 2015.

[3] Y. Kurihara, T. Shishido, K. Oku et al., "Polymyositis associated with autoimmune hepatitis, primary biliary cirrhosis, and autoimmune thrombocytopenic purpura," Modern Rheumatology, vol. 21, no. 3, pp. 325-329, 2011.

[4] G. S. Hatzis, G. E. Fragoulis, A. Karatzaferis, I. Delladetsima, C. Barbatis, and H. M. Moutsopoulos, "Prevalence and longterm course of primary biliary cirrhosis in primary Sjögren's syndrome," Journal of Rheumatology, vol. 35, no. 10, pp. 2012-2016, 2008.

[5] R. Hua, H. Wu, X. W. Zhang, and Y. W. Sun, "Probable catastrophic antiphospholipid syndrome complicated with primary sclerosing cholangitis," Journal of Digestive Diseases, vol. 13, no. 11, pp. 601-603, 2012.

[6] G. W. Wong, T. Yeong, D. Lawrence et al., "Concurrent extrahepatic autoimmunity in autoimmune hepatitis: implications for diagnosis, clinical course and long-term outcomes," Liver International, 2016.

[7] G. W. Wong and M. A. Heneqhan, "Association of extrahepatic manifestations with autoimmune hepatitis," Digestive Diseases, vol. 33, supplement 2, pp. 25-35, 2015. 
[8] N. Bach and J. A. Odin, "Primary biliary cirrhosis: a Mount Sinai perspective," The Mount Sinai Journal of Medicine, vol. 70, no. 4, pp. 242-250, 2003.

[9] P. L. Bittencourt, A. Q. Farias, G. Porta et al., "Frequency of concurrent autoimmune disorders in patients with autoimmune hepatitis: effect of age, gender, and genetic background," Journal of Clinical Gastroenterology, vol. 42, no. 3, pp. 300-305, 2008.

[10] M. Kmieciak Le Corguillé, P. Rocher, C. Eugène et al., "Autoimmune hepatitis, acute pancreatitis, mixed connective tissue disease and Sjögren's syndrome. A case report," Gastroenterologie Clinique et Biologique, vol. 27, no. 8-9, pp. 840-841, 2003.

[11] M. J. Kaplan and R. W. Ike, "The liver is a common non-exocrine target in primary Sjögren's syndrome: a retrospective review," BMC Gastroenterology, vol. 2, article 21, 2002.

[12] S. Branger, N. Schleinitz, V. Veit et al., "Auto-immune hepatitis and antiphospholipids," Revue de Medecine Interne, vol. 28, no. 4, pp. 218-224, 2007.

[13] A. Floreani, I. Franceschet, N. Cazzagon et al., "Extrahepatic autoimmune conditions associated with primary biliary cirrhosis," Clinical Reviews in Allergy and Immunology, vol. 48, no. 2-3, pp. 192-197, 2015.

[14] J. K. Karp, E. K. Akpek, and R. A. Anders, "Autoimmune hepatitis in patients with primary Sjögren's syndrome: a series of two-hundred and two patients," International Journal of Clinical and Experimental Pathology, vol. 3, no. 6, pp. 582-586, 2010.

[15] T. Matsumoto, S. Kobayashi, H. Shimizu et al., "The liver in collagen diseases: pathologic study of 160 cases with particular reference to hepatic arteritis, primary biliary cirrhosis, autoimmune hepatitis and nodular regenerative hyperplasia of the liver," Liver, vol. 20, no. 5, pp. 366-373, 2000.

[16] C. Selmi, M. De Santis, and M. E. Gershwin, "Liver involvement in subjects with rheumatic disease," Arthritis Research \& Therapy, vol. 13, no. 3, article 226, 2011.

[17] D. C. Rockey, S. H. Caldwell, Z. D. Goodman, R. C. Nelson, and A. D. Smith, "Liver biopsy," Hepatology, vol. 49, no. 3, pp. 1017$1044,2009$.

[18] E. A. Aleksandrova, E. Z. Burnevich, and E. A. Arion, "Systemic manifestations of primary sclerosing cholangitis," Klinicheskaia Meditsina, vol. 91, no. 4, pp. 38-42, 2013.

[19] L. Wang, F.-C. Zhang, H. Chen et al., "Connective tissue diseases in primary biliary cirrhosis: a population-based cohort study," World Journal of Gastroenterology, vol. 19, no. 31, pp. 51315137, 2013.

[20] S. Abraham, S. Begum, and D. Isenberg, "Hepatic manifestations of autoimmune rheumatic diseases," Annals of the Rheumatic Diseases, vol. 63, no. 2, pp. 123-129, 2004.

[21] W. I. Youssef and A. S. Tavill, "Connective tissue diseases and the liver," Journal of Clinical Gastroenterology, vol. 35, no. 4, pp. 345-349, 2002.

[22] M. Ramos-Casals, Roberto-Perez-Alvarez, C. Diaz-Lagares, M.J. Cuadrado, and M. A. Khamashta, "Autoimmune diseases induced by biological agents: a double-edged sword?" Autoimmunity Reviews, vol. 9, no. 3, pp. 188-193, 2010.

[23] K. Visser, W. Katchamart, E. Loza et al., "Multinational evidence-based recommendations for the use of methotrexate in rheumatic disorders with a focus on rheumatoid arthritis: integrating systematic literature research and expert opinion of a broad international panel of rheumatologists in the $3 \mathrm{E}$ Initiative," Annals of the Rheumatic Diseases, vol. 68, no. 7, pp. 1086-1093, 2009.
[24] G. M. Hirschfield, X. Liu, C. Xu et al., "Primary biliary cirrhosis associated with HLA, IL $12 A$, and IL12RB2 variants," The New England Journal of Medicine, vol. 360, pp. 2544-2555, 2009.

[25] C. Selmi, P. Invernizzi, M. Zuin, M. Podda, and M. E. Gershwin, "Genetics and geoepidemiology of primary biliary cirrhosis: following the footprints to disease etiology," Seminars in Liver Disease, vol. 25, no. 3, pp. 265-280, 2005.

[26] C. Selmi, M. J. Mayo, N. Bach et al., "Primary biliary cirrhosis in monozygotic and dizygotic twins: genetics, epigenetics, and environment," Gastroenterology, vol. 127, no. 2, pp. 485-492, 2004.

[27] Y. Liu, J. Yu, Z. Oaks et al., "Liver injury correlates with biomarkers of autoimmunity and disease activity and represents an organ system involvement in patients with systemic lupus erythematosus," Clinical Immunology, vol. 160, no. 2, pp. 319327,2015

[28] M. H. El-Shabrawi and M. I. Farrag, "Hepatic manifestations in juvenile systemic lupus erythematosus," Recent Patents on Inflammation and Allergy Drug Discovery, vol. 8, no. 1, pp. 3640, 2014.

[29] M. De Santis, C. Crotti, and C. Selmi, "Liver abnormalities in connective tissue diseases," Best Practice and Research: Clinical Gastroenterology, vol. 27, no. 4, pp. 543-551, 2013.

[30] Y. Akiyama, M. Tanaka, M. Takeishi, D. Adachi, A. Mimori, and T. Suzuki, "Clinical, serological and genetic study in patients with CREST syndrome," Internal Medicine, vol. 39, no. 6, pp. 451-456, 2000.

[31] H. Y. Ju, J. Y. Jang, S. W. Jeong et al., "The clinical features of drug-induced liver injury observed through liver biopsy: focus on relevancy to autoimmune hepatitis," Clinical and Molecular Hepatology, vol. 18, no. 2, pp. 213-218, 2012.

[32] A. Hewagama and B. Richardson, "The genetics and epigenetics of autoimmune diseases," Journal of Autoimmunity, vol. 33, no. 1, pp. 3-11, 2009.

[33] C. Selmi, P. L. Meroni, and M. E. Gershwin, "Primary biliary cirrhosis and Sjögren's syndrome: autoimmune epithelitis," Journal of Autoimmunity, vol. 39, no. 1-2, pp. 34-42, 2012.

[34] E. Savarino, M. Furnari, N. de Bortoli et al., "Gastrointestinal involvement in systemic sclerosis," Presse Medicale, vol. 43, no. 10, pp. 279-291, 2014.

[35] I. R. Mackay, "Clustering and commonalities among autoimmune diseases," Journal of Autoimmunity, vol. 33, no. 3-4, pp. 170-177, 2009.

[36] A. Martínez, J. Varadé, A. Márquez et al., "Association of the STAT4 gene with increased susceptibility for some immunemediated diseases," Arthritis \& Rheumatism, vol. 58, no. 9, pp. 2598-2602, 2008.

[37] T. S. Rodríguez-Reyna and D. Alarcón-Segovia, "Overlap syndromes in the context of shared autoimmunity," Autoimmunity, vol. 38, no. 3, pp. 219-223, 2005.

[38] C. Selmi and M. E. Gershwin, "The role of environmental factors in primary biliary cirrhosis," Trends in Immunology, vol. 30, no. 8, pp. 415-420, 2009.

[39] A. Castiella, E. Zapata, M. I. Lucena, and R. J. Andrade, "Druginduced autoimmune liver disease: a diagnostic dilemma of an increasingly reported disease," World Journal of Hepatology, vol. 6, no. 4, pp. 160-168, 2014.

[40] S. Rodrigues, S. Lopes, F. Magro et al., "Autoimmune hepatitis and anti-tumor necrosis factor alpha therapy: a single center report of 8 cases," World Journal of Gastroenterology, vol. 21, no. 24, pp. 7584-7588, 2015. 
[41] E. Björnsson, J. Talwalkar, S. Treeprasertsuk et al., "Druginduced autoimmune hepatitis: clinical characteristics and prognosis," Hepatology, vol. 51, no. 6, pp. 2040-2048, 2010.

[42] A. Suzuki, E. M. Brunt, D. E. Kleiner et al., "The use of liver biopsy evaluation in discrimination of idiopathic autoimmune hepatitis versus drug-induced liver injury," Hepatology, vol. 54, no. 3, pp. 931-939, 2011.

[43] P. Muratori, A. Fabbri, C. Lalanne, M. Lenzi, and L. Muratori, "Autoimmune liver disease and concomitant extrahepatic autoimmune disease," European Journal of Gastroenterology and Hepatology, vol. 27, no. 10, pp. 1175-1179, 2015.

[44] C. Rigamonti, L. M. Shand, M. Feudjo et al., "Clinical features and prognosis of primary biliary cirrhosis associated with systemic sclerosis," Gut, vol. 55, no. 3, pp. 388-394, 2006.

[45] E. F. Remmers, R. M. Plenge, A. T. Lee et al., "STAT4 and the risk of rheumatoid arthritis and systemic lupus erythematosus," The New England Journal of Medicine, vol. 357, no. 10, pp. 977-986, 2007.

[46] R. Chapman, J. Fevery, A. Kalloo et al., "Diagnosis and management of primary sclerosing cholangitis," Hepatology, vol. 51, no. 2, pp. 660-678, 2010.

[47] S. N. Dastis, D. Latinne, C. Sempoux, and A. P. Geubel, "Ulcerative colitis associated with IgG4 cholangitis: Similar features in two HLA identical siblings," Journal of Hepatology, vol. 51, no. 3, pp. 601-605, 2009.

[48] Y. Goto, Y. Kurashima, and H. Kiyono, "The gut microbiota and inflammatory bowel disease," Current Opinion in Rheumatology, vol. 27, no. 4, pp. 388-396, 2015.

[49] M. A. Kriegel, "Self or non-self? The multifaceted role of the microbiota in immune-mediated diseases," Clinical Immunology, vol. 159, no. 2, pp. 119-121, 2015.

[50] G. M. Hirschfield, E. J. Heathcote, and M. E. Gershwin, "Pathogenesis of cholestatic liver disease and therapeutic approaches," Gastroenterology, vol. 139, no. 5, pp. 1481-1496, 2010.

[51] C. Selmi, I. R. Mackay, and M. E. Gershwin, "The autoimmunity of primary biliary cirrhosis and the clonal selection theory," Immunology \& Cell Biology, vol. 89, no. 1, pp. 70-80, 2011.

[52] M. E. Gershwin and I. R. Mackay, "The causes of primary biliary cirrhosis: convenient and inconvenient truths," Hepatology, vol. 47, no. 2, pp. 737-745, 2008.

[53] T. Matsumoto, T. Morizane, Y. Aoki et al., "Autoimmune hepatitis in primary Sjögren's syndrome: pathological study of the livers and labial salivary glands in 17 patients with primary Sjögren's syndrome," Pathology International, vol. 55, no. 2, pp. 70-76, 2005.

[54] P. Obermayer-Straub, J. Perheentupa, S. Braun et al., "Hepatic autoantigens in patients with autoimmune polyendocrinopathy-candidiasis-ectodermal dystrophy," Gastroenterology, vol. 121, no. 3, pp. 668-677, 2001. 


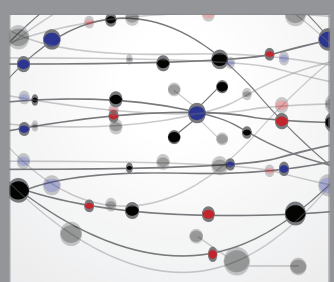

The Scientific World Journal
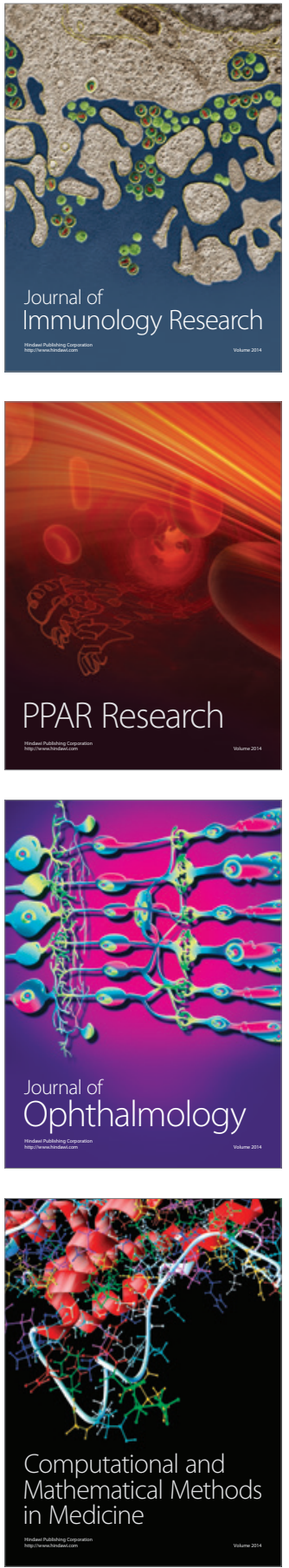

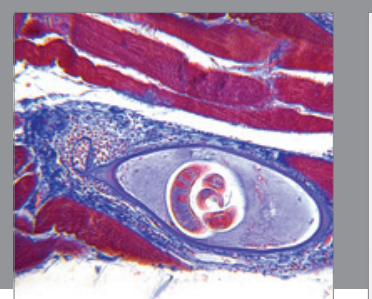

Gastroenterology Research and Practice
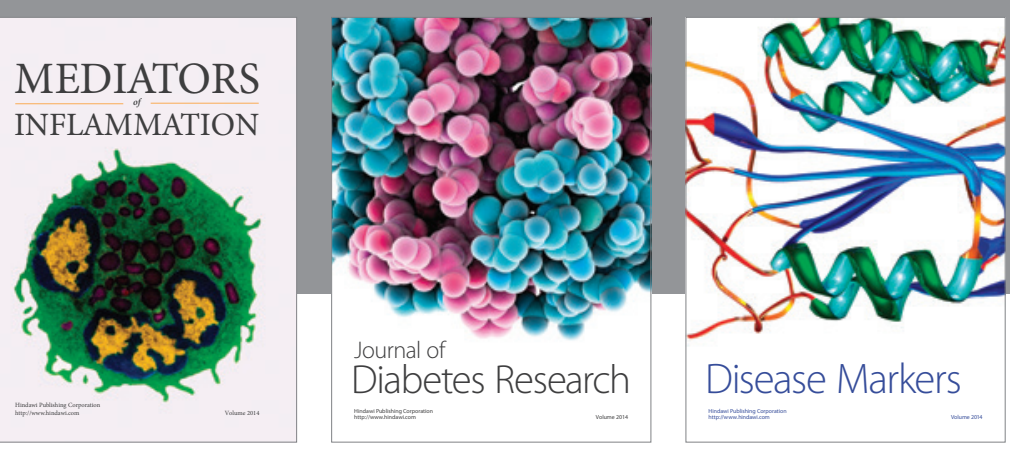

Disease Markers

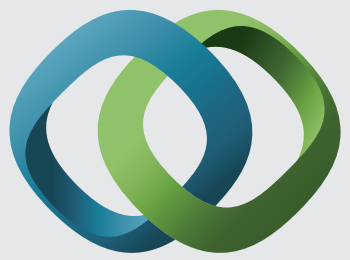

\section{Hindawi}

Submit your manuscripts at

https://www.hindawi.com
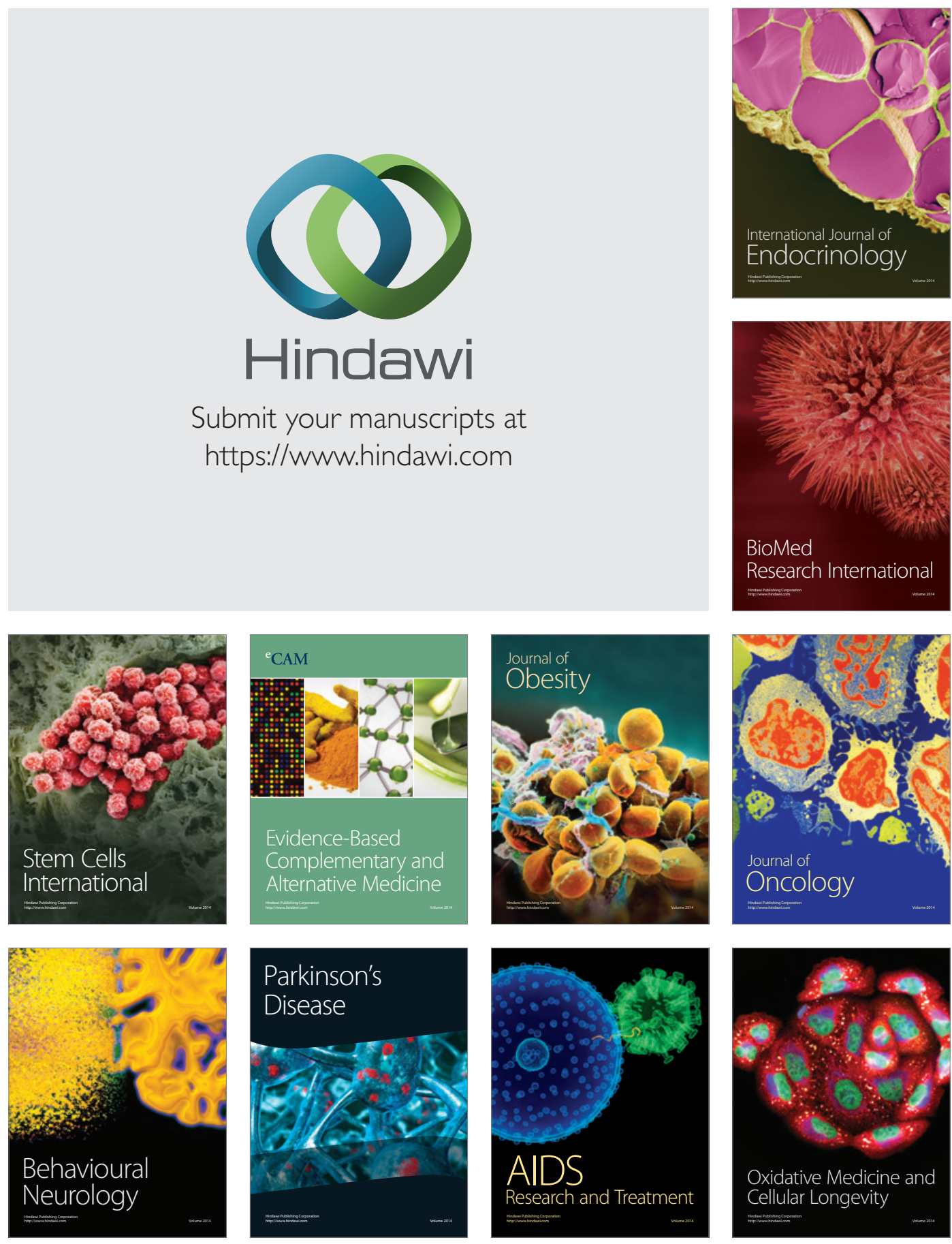